\title{
BRANCHING PROCESSES WITH DEPENDENCE BUT HOMOGENEOUS GROWTH ${ }^{1}$
}

\author{
By Peter JAgers \\ Chalmers University of Technology
}

\begin{abstract}
A (general) branching process, where individuals need not reproduce independently, satisfies a homogeneous growth condition if, vaguely, one would not expect the progeny from any one individual to make out more than its proper fraction of the whole population at any time in the future. This notion is made precise, and it is shown how it entails classical Malthusian growth in supercritical cases, in particular for population size-dependent Bienaymé-Galton-Watson and Markov branching processes, and for nondecreasing age-dependent processes with continuous life span distributions.
\end{abstract}

1. Introduction. It is easy to envisage general branching processes with possible interaction between individuals, instead of the classical independence. If the interaction stabilizes during supercritical growth, so that in the limit individuals reproduce independently, like members of a classical, general multitype and supercritical branching population, then a coupling device can be used to establish Malthusian, that is, exponential, growth.

Indeed, assume that individual reproductions in the limiting infinite population tend not to exceed those of finite populations. Then the coupling can be constructed through imaginary abortions so that the resulting, thinned reproduction process equals what would have been the case, had the population been infinite [Jagers (1997)]. The population of nonaborted individuals, from any time onwards, must asymptotically grow exponentially (or die out), and if no abortions occur after a finite (but random) time, the same must be ultimately true of the original, not thinned population.

A particularly lucid case is that of population size dependence in single-type populations. If $m(n)$ denotes the expected offspring per individual in an $n$ size generation and $m=m(\infty)$ the same in an imaginary infinite population, then in many cases the coupling will be successful—and Malthusianness thus established-if

$$
\sum\{m(n)-m\}<\infty
$$

Received August 1998; revised January 1999.

${ }^{1}$ This work is part of the Bank of Sweden Tercentenary Foundation Project Dependence and Interaction in Stochastic Population Dynamics.

AMS 1991 subject classifications. Primary 60J80; secondary 60F25, 92A15.

Key words and phrases. Branching processes, population dynamics, cell kinetics, population size dependence. 
However, as pointed out [Jagers (1997)], exponential growth can occur even in cases where this coupling fails. In particular Klebaner (1984, 1985, 1989) managed to show that

$$
\sum\{m(n)-m\} / n<\infty
$$

essentially suffices for Bienaymé-Galton-Watson processes to exhibit Malthusian growth $\sim m^{n}$.

For a binary splitting cell model, Gyllenberg and Webb (1990) arrived at the same type of main condition in a deterministic context.

The purpose of the present paper is to establish Klebaner-type criteria for simple and certain age-dependent branching processes, thus replacing the, in that regard, rather preliminary attempts made in Jagers (1997).

Basic in this is the concept of symmetric or homogeneous growth [Jagers (1997)] crucially linked to the factor $1 / n$ in Klebaner's formula.

Consider a general (possibly multitype) branching population. Its probability space can be constructed explicitly in the traditional Ulam-Harris or Neveu (1986) tree manner, only the probability measure is not required to have the (conditional) independence properties of classical branching. However, it must be required that only finitely many individuals are born in finite time intervals, so that individuals can be numbered as they are born into the population, arbitrarily for individuals born together but always so that a mother precedes her daughter, $X_{1}, X_{2}, X_{3}, \ldots$.

The population size at time $t, z_{t}^{\chi}$, can be generally defined by some characteristic $\chi$ [cf. Jagers (1989)], though a certain care has to be exerted in allowing the population as a whole to affect the measure used. We shall only consider simple size processes like those alive, $\left\{z_{t}\right\}$, or $\left\{y_{t}\right\}$, the total population of all those born. In the specific cases we shall go into deeper, life spans of different individuals will be presumed independent of one another and of everything else. In a model where dependence is on population size, the latter may thus influence reproductions but not life spans.

Denote the birth time of an individual $x$ by $\tau_{x}$ and $x$ 's daughter process, the number of individuals stemming from $x$ and alive at $t$ by $z_{t}(x)$. Further, write $y_{t}(x)$ for all those stemming from $x$ born by $t$, alive or not. For simplicity, write $\tau_{i}$ instead of $\tau_{X_{i}}$, even though that is ambiguous with individuals labelled in the Ulam-Harris manner. Let $\mathscr{G}_{x}$ be the $\sigma$-algebra containing all information about matters that occurred up to $\tau_{x}$, that is, $\mathscr{G}_{\tau_{x}}$, and abbreviate $\mathscr{G}_{X_{i}}$ further into $\mathscr{G}_{i}$. Interpret $0 / 0$ and $1 / z_{\infty}$ as zero, and keep in mind that the latter convention means that sums over elements divided by $z_{\tau_{x}}$ will only be taken over those where $\tau_{x}<\infty$, finitely many in case of extinction. To avoid uninteresting complications, we assume that a daughter cannot be born simultaneously with her mother.

DEFINITION 1. A branching process with possible dependence between individuals is said to grow homogeneously (or to exhibit symmetric growth) if 
there is a constant $K$ such that

$$
\mathbb{E}\left[\frac{z_{t-\tau_{x}}(x)}{z_{t}} \mid \mathscr{G}_{x}\right] \leq \frac{K}{z_{\tau_{x}}} .
$$

for all individuals $x$ and $t \geq \tau_{x}$.

This means that the expected fraction of the future subpopulations, stemming from anyone of the $z_{t}$ alive at any time $t$, are all of the same order of magnitude.

The obvious example of such a process is a Bienaymé-Galton-Watson process with population size dependence. Since the reproductions of all individuals in the same, say $k$ th, generation are affected by the same population size and are otherwise independent, symmetry implies that

$$
\mathbb{E}\left[\frac{z_{n-k}(x)}{z_{n}} \mid \mathscr{G}_{x}\right]=\frac{1}{z_{k}},
$$

for any $x$ with $\tau_{x}=k$. More about that in the next section.

Now we shall give the general result about Malthusianness for homogeneously growing populations, whose reproductions approach those of an independent individual Malthusian population from above.

Thus, denote the reproductions of any individual $x$ by $\xi_{x}$. In the general case this is a point process telling at which ages $x$ begets children of what types from a general type space $S$. For single-type, age-dependent or Galton-Watson processes it reduces to a random variable, telling us how many children $x$ splits into at death. Similarly, let $\eta_{x}$ be the reproduction process of a general Malthusian branching population with the finite reproduction kernel $\mu$ (defining the Malthusian parameter $\alpha$, stable type distribution $\pi$, and fitness $h$ ). Interpret "Malthusian branching population" to mean that the classical supercritical convergence theorem should hold [Jagers (1989)]. Assume that for any measurable set $A$ of types and age interval $B, \xi_{x}(A \times B) \geq \eta_{x}(A \times B)$ in distribution given $\mathscr{G}_{x}$.

Then, by Strassen's theorem [Lindvall (1992)] the two processes can be coupled through imaginary abortions: $\eta_{x}$, can be thought of as arising from $\xi_{x}$ through deletion of some children. Again abusing notation somewhat, we let $\xi_{x}, \eta_{x}$ stand for a coupled version and write $\delta_{x}$ to indicate abortion or not of $x$, so that $\delta_{x}=1$ if and only if $x$ is an atom of $\xi_{m x}$ but not of $\eta_{m x}, m x$ denoting $x$ 's mother in the Ulam-Harris tree.

We now think of $\mathscr{G}_{x}$ as referring to the process as defined on this new space. Note that in a splitting process $\xi_{m x} \in \mathscr{G}_{x}$, but generally this need not be the case. However, except for this general section we shall only deal with splitting populations in this paper, that is, populations where childbearing can only occur once in a mother's life, at her death. Therefore we refrain from further discussion of the measurability aspects of the problem. However, we shall assume that the coupling has been so constructed that the future of the actual process from any $\tau_{x}$ onwards is independent of $\eta_{m x}$, given $\mathscr{G}_{x}$ and $\xi_{m x}$. 
THEOREM 2. Let $\left\{z_{t}\right\}$ be a general branching process with symmetric growth. Assume that there is a general supercritical Malthusian branching population with minorizing reproductions as above. Assume that $w_{t}:=$ $\exp (-\alpha t) z_{t}$ is tight. Then,

$$
\sum_{i} \mathbb{E}\left[\delta_{i} / z_{\tau_{i}}\right]<\infty
$$

implies that $w_{t}$ tends in probability to some finite $w \geq 0$, not identically zero on the set of nonextinction.

PROOF. The proof is from Jagers (1997), where there is also more about the coupling and general construction. It is reproduced here for completeness, and since there are slight differences in setup.

Write tildes over processes that count only nonaborted individuals, that is, individuals born according to the $\eta$ reproduction processes. Thus, $\tilde{z}_{t-\tau_{x}}(x)$ stands for the process starting from the individual $x$ in the actual population at time $\tau_{x}$ but thinned by the imaginary abortions. Similarly, $\tilde{w}_{t-\tau_{x}}(x):=$ $\exp \left(-\alpha\left(t-\tau_{x}\right)\right) \tilde{z}_{t-\tau_{x}}(x)$ and so on.

For any $u, z_{t}^{u}$ records at time $t+u$ the number of all living individuals born up to $u$ but only living individuals not stemming from an aborted individual after $u$. If $\delta_{x u}=0$ precisely if $x$ stems from an $x^{\prime}$ with $\tau_{x^{\prime}}>u$ and $\delta_{x^{\prime}}=1$, and $\delta_{x u}=1$ otherwise, and $\chi_{x}(a)$ indicates whether $x$ is alive or not at age $a$, thus

$$
z_{t}^{u}=\sum_{\tau_{x} \leq t+u} \delta_{x u} \chi_{x}\left(t+u-\tau_{x}\right) .
$$

In analogy with other notation $w_{t}^{u}:=\exp (-\alpha(t+u)) z_{t}^{u}$.

Clearly,

$$
0 \leq z_{t+u}-z_{t}^{u} \leq \sum_{\tau_{x}>u} \delta_{x} z_{t+u-\tau_{x}}(x)
$$

and

$$
\left|w_{t+u}-w_{t}^{u}\right| \leq w_{t+u} \sum_{\tau_{x}>u} \delta_{x} \frac{z_{t+u-\tau_{x}}(x)}{z_{t+u}} .
$$

Thus for any $\varepsilon^{\prime}, v>0$ the homogeneous growth condition yields

$$
\mathbb{P}\left(\left|w_{t+u}-w_{t}^{u}\right|>\varepsilon^{\prime}, w_{t+u} \leq v\right) \leq\left(v / \varepsilon^{\prime}\right) K \mathbb{E}\left[\sum_{\tau_{x}>u} \delta_{x} / z_{\tau_{x}}\right]
$$

for all $t$ and starting types $s \in S$. But

$$
\left|w_{t+u}-w_{t^{\prime}+u}\right| \leq\left|w_{t+u}-w_{t}^{u}\right|+\left|w_{t}^{u}-w_{t^{\prime}}^{u}\right|+\left|w_{t^{\prime}+u}-w_{t^{\prime}}^{u}\right| .
$$

Since, with $I_{u}:=\left\{x ; \tau_{m x} \leq t<\tau_{x}<\infty\right\}$,

$$
w_{t}^{u}=\sum_{x \in I_{u}} \exp \left(-\alpha \tau_{x}\right) \tilde{w}_{t+u-\tau_{x}}(x) \rightarrow \sum_{x \in I_{u}} \exp \left(-\alpha \tau_{x}\right) \tilde{w}(x),
$$


as $t \rightarrow \infty$, the tilded daughter processes being independent individual supercritical branching processes with Malthusian parameter $\alpha$, it follows that

$$
\lim _{t, t^{\prime} \rightarrow \infty} \mathbb{P}\left(\left|w_{t}^{u}-w_{t^{\prime}}^{u}\right|>\varepsilon^{\prime}\right)=0 .
$$

Hence,

$$
\limsup _{t, t^{\prime} \rightarrow \infty} \mathbb{P}\left(\left|w_{t+u} \wedge v-w_{t^{\prime}+u} \wedge v\right|>\varepsilon^{\prime}\right) \leq 2\left(v / \varepsilon^{\prime}\right) K \mathbb{E}\left[\sum_{\tau_{x}>u} \delta_{x} / z_{\tau_{x}}\right] .
$$

Since $u$ can be chosen so as to render the right-hand side arbitrarily small, the convergence in probability follows by completeness and tightness.

Now define the (conditional) abortion probability

$$
\varepsilon_{x}:=\mathbb{E}\left[\delta_{x} \mid \mathscr{G}_{x-}\right],
$$

where $\mathscr{G}_{x-}$ is $\mathscr{G}_{\tau_{x}-}$, the $\sigma$-algebra of events strictly preceding $\tau_{x}$. We write $\varepsilon_{i}$ for $\varepsilon_{X_{i}}$ and $\mathscr{I}_{i-}$ for $\mathscr{G}_{X_{i}-}$. If $\varepsilon_{x}$ turns out to be a function of population size immediately before $\tau_{x}, z_{\tau_{x}-}$, the process is said to be population size dependent.

COROLlaRY 3. The summation convergence condition of Theorem 2 can be replaced by

$$
\sum \mathbb{E}\left[\varepsilon_{i} / z_{\tau_{i}}\right]<\infty
$$

PRoOF. Write $m_{i}$ for $X_{i}$ 's mother and $r_{i}$ for her rank among her sisters, and let $r_{i} \notin \eta_{m_{i}}$ mean that the individual would not have been born, had the population been infinite (and hence since she is born into the actual one that she is aborted). With superscripts denoting conditional expectation then,

$$
\begin{aligned}
\mathbb{E}^{\mathscr{G}_{i-}\left[\delta_{i} / z_{\tau_{i}}\right]} & =\mathbb{P}^{\mathscr{G}_{i-}}\left(\delta_{i}=1\right) \mathbb{E}^{\mathscr{G}_{i-}}\left[1 / z_{\tau_{i}} \mid \delta_{i}=1\right. \\
& =\varepsilon_{i} \mathbb{E}^{\mathscr{G}_{i-}}\left[1 / z_{\tau_{i}} \mid \tau_{i}<\infty, r_{i} \notin \eta_{m_{i}}\right] .
\end{aligned}
$$

Since $z_{\tau_{i}}$ is independent of $\eta_{m_{i}}$, given $\mathscr{C}_{i-}$ and $\tau_{i}<\infty$, and $1 / z_{\tau_{i}}=0$ if $\tau_{i}=\infty$, indeed,

$$
\begin{aligned}
\mathbb{E}^{\mathscr{G}_{i-}}\left[\delta_{i} / z_{\tau_{i}}\right] & =\varepsilon_{i} \mathbb{E}^{\mathscr{G}_{i-}}\left[1 / z_{\tau_{i}} \mid \tau_{i}<\infty\right]=\varepsilon_{i} \mathbb{E}^{\mathscr{G}_{i-}}\left[1 / z_{\tau_{i}}\right] / \mathbb{P}^{\mathscr{G}_{i-}}\left(\tau_{i}<\infty\right) \\
& =\varepsilon_{i} \mathbb{E}^{\mathscr{G}_{i-}}\left[1 / z_{\tau_{i}}\right],
\end{aligned}
$$

as for any $t,\left\{\tau_{i}<\infty\right\} \cap\left\{\tau_{i} \leq t\right\} \in \mathscr{G}_{t}$, so that $\tau_{i}$ is measurable with respect to $\mathscr{G}_{i-}$, and thus $\mathbb{P}^{\mathscr{G}_{i-}}\left(\tau_{i}<\infty\right)=1$ on the set $\tau_{i}<\infty$. But only such sets matter in the expectations summed.

COROLLARY 4. Consider a single-type splitting population with continuously distributed life spans. Write $T_{1}<T_{2}<\cdots$ for the successive splitting times, $m_{x}:=\mathbb{E}\left[\xi_{x} \mid \mathscr{G}_{x-}\right], \xi_{x}$ now simply denoting $x$ 's number of children, and 
$m:=\mathbb{E}\left[\eta_{x} \mid \mathscr{G}_{x-}\right]=\mathbb{E}\left[\eta_{x}\right]$. Then,

$$
\sum \mathbb{E}\left[\varepsilon_{i} / z_{\tau_{i}}\right]=\sum \mathbb{E}\left[\left(m_{i}-m\right) / z_{T_{i}}\right],
$$

$m_{i}$ being short for $m$ indexed by the individual splitting at $T_{i}$.

In particular, if the process is population size dependent, then $m_{i}=m\left(z_{T_{i}}\right)$.

The proof of this is straightforward (and the corollary can easily be generalized to cases where many mothers may give birth simultaneously, as in the discrete time situation below).

2. The Bienaymé-Galton-Watson case. A population size- (or state-) dependent (Bienaymé-)Galton-Watson process is a discrete time branching process $\left\{z_{n}\right\}$, where given the history $\mathscr{A}_{n}$ during the first $n$ generations, $z_{n+1}$ is the sum of $z_{n}$ i.i.d. nonnegative integer valued random variables, whose distribution $\left\{p_{k}\left(z_{n}\right)\right\}$ is determined by the present population size, $z_{n}$. The minorization condition can be expressed as

$$
\sum_{k \geq j} p_{k}(n) \geq \sum_{k \geq j} p_{k}
$$

for all $j$ and $n, p_{k}=p_{k}(\infty)$ being thought of as the reproduction law "if the population were infinite." The latter is supposed to define a supercritical Malthusian Galton-Watson process,

$$
m=m(\infty)=\sum_{k} k p_{k}>1, \quad \sum_{k} k(\log k) p_{k}<\infty .
$$

Similarly, write $m(n):=\sum_{k} k p_{k}(n)$. A Galton-Watson process is sometimes called nonlattice or aperiodic if the group generated by the possible transitions of the process, viewed as a Markov chain, is all of $\mathbb{Z}$ [cf. Dubuc (1970) or Athreya and Ney (1972), page 93].

In the present case $\xi_{x}$ is just a random variable, $x$ 's number of children and $\eta_{x}$ the number retained. Hence, if $x$ belongs to the $n$th generation, $\xi_{x} \mid \mathscr{A}_{n} \sim$ $\left\{p_{k}\left(z_{n}\right)\right\}$ and $\eta_{x} \mid \mathscr{A}_{n} \sim\left\{p_{k}\right\}$. Since

$$
\begin{aligned}
\delta_{x j} & =1_{\left\{\xi_{x} \geq j\right\}}-1_{\left\{\eta_{x} \geq j\right\}}, \\
\varepsilon_{x j} & =\sum_{k \geq j} p_{k}\left(z_{n}\right)-\sum_{k \geq j} p_{k}
\end{aligned}
$$

and

$$
\sum_{j} \varepsilon_{x j}=m\left(z_{n}\right)-m
$$

THEOREM 5. A population size-dependent Galton-Watson process is symmetrically dependent. Make the minorization assumption above, assume that $m(n) \searrow m>1$ and

$$
\sum_{n}\{m(n)-m\} / n<\infty .
$$


Then $\left\{w_{n}:=z_{n} / m^{n}\right\}$ is tight. If further $\left\{z_{n}^{\infty}\right\}$ is aperiodic and $\sum k(\log k)^{2} p_{k}<$ $\infty$, then $w_{n}$ has a nontrivial limit in probability.

LEMMA 6. Let $\left\{z_{n}\right\}$ be an aperiodic Galton-Watson process with $\sum k p_{k}=$ $m>1$ and $\sum k(\log k)^{2} p_{k}<\infty$. Then the Greens function

$$
G(1, k):=\sum_{n} \mathbb{P}\left(z_{n}=k\right)
$$

is $O(1 / k)$, as $k \rightarrow \infty$.

This follows from Theorem 9 in Dubuc (1970); compare also Athreya and Ney [(1972), page 93]. The theorem itself is due to Klebaner (1985), with almost sure convergence in the conclusion and a condition $(\mathrm{C})$, a sort of generalized or uniform $x \log x$, replacing our $x \log ^{2} x$-condition. For a thorough classification of behavior of models described by recurrence relations, see Keller, Kersting and Rösler (1987).

PROOF OF THE THEOREM. With $y_{k}$ still denoting the total population, $\tau_{i}=$ $k \Leftrightarrow y_{k-1}<i \leq y_{k}$. Thus, if $\mathscr{g}_{k}$ denotes the realized $k$ th generation, $\{x \in$ $\left.N^{k} ; \tau_{x}<\infty\right\}$ in Ulam-Harris notation, then

$$
\begin{aligned}
\sum \varepsilon_{i} / z_{\tau_{i}} & =\sum_{k} \sum_{i=y_{k-1}+1}^{y_{k}} \varepsilon_{i} / z_{k}, \\
\sum_{k} \sum_{x \in \mathscr{O}_{k-1}} \sum_{j} \varepsilon_{x j} / z_{k} & =\sum_{k} \sum_{x \in \mathscr{O}_{k-1}}\left\{m\left(z_{k-1}\right)-m\right\} / z_{k} \\
& =\sum_{k} z_{k-1}\left\{m\left(z_{k-1}\right)-m\right\} / z_{k} .
\end{aligned}
$$

Writing $g(n):=m(n)-m$, we can conclude that

$$
\sum \mathbb{E}\left[\varepsilon_{i} / z_{\tau_{i}}\right]=\sum \mathbb{E}\left[z_{k-1} g\left(z_{k-1}\right) / z_{k}\right]
$$

However, given $\mathscr{A}_{k-1}, z_{k}$ is larger than or equal to a variable $U$ which is binomial $z_{k-1}, 1-p_{0}\left(z_{k-1}\right)$. Since if $Z$ is $\operatorname{Bin}(n, p)$,

$$
\begin{aligned}
\mathbb{E}\left[\frac{1}{Z+1}\right] & =\frac{1-(1-p)^{n+1}}{(n+1) p}, \\
\mathbb{E}\left[1 / z_{k} ; z_{k} \geq 1 \mid \mathscr{A}_{k-1}\right] & \leq \mathbb{E}[1 / U ; U \geq 1] \\
& \leq \mathbb{E}[2 /(U+1)] \leq 2 /\left(z_{k-1}\left\{1-p_{0}\left(z_{k-1}\right)\right\}\right) \\
& \leq 2 /\left(z_{k-1}\left(1-p_{0}\right)\right) .
\end{aligned}
$$

Hence,

$$
\sum \mathbb{E}\left[\varepsilon_{i} / z_{\tau_{i}}\right] \leq \sum C \mathbb{E}\left[g\left(z_{k-1}\right) ; z_{k-1} \geq 1\right],
$$

for some constant $C$. But by assumption $g(k)=m(k)-m$ does not increase and $\sum g(k) / k<\infty$. Further, $z_{n} \stackrel{d}{\geq} z_{n}^{\infty}$, where the latter is Galton-Watson with 
reproduction distribution $\left\{p_{k}\right\}$, "as if the population were infinite all the time." By Lemma 6 , with $G(1, k)$ the Greens function of $\left\{z_{n}^{\infty}\right\}$, thus

$$
\begin{aligned}
\sum_{n} \mathbb{E}\left[g\left(z_{n}\right) ; z_{n} \geq 1\right] & \leq \sum_{n} \mathbb{E}\left[g\left(z_{n}^{\infty}\right) ; z_{n}^{\infty} \geq 1\right] \\
& =\sum_{n} \sum_{k} g(k) \mathbb{P}\left(z_{n}^{\infty}=k\right)=\sum_{k} g(k) G(1, k) \\
& \leq c \sum_{k} g(k) / k<\infty,
\end{aligned}
$$

where $c$ is the ordo constant from Lemma 6 . It follows that $\sum \mathbb{E}\left[\varepsilon_{i} / z_{\tau_{i}}\right]$ converges, as required by Corollary 3 .

It remains to check the asserted tightness of $\left\{w_{n}\right\}=\left\{z_{n} / m^{n}\right\}$. We shall show that $\left\{\mathbb{E}\left[w_{n}\right]\right\}$ is bounded. By the assumptions on $g$, or rather $m(n)-m$, there exists a $G \geq g$ which does not increase, is such that $x G(x)$ is nondecreasing and concave on $\mathbb{R}_{+}$and satisfies $\sum G(n) / n<\infty$. In terms of this $G$,

$$
\mathbb{E}\left[w_{n} \mid \mathscr{A}_{n-1}\right]=w_{n-1}+z_{n-1} g\left(z_{n-1}\right) / m^{n} \leq w_{n-1}+z_{n-1} G\left(z_{n-1}\right) / m^{n}
$$

for any $n$. Hence,

$$
\begin{aligned}
\mathbb{E}\left[w_{n}\right] & \leq \mathbb{E}\left[w_{n-1}\right]+\mathbb{E}\left[z_{n-1} G\left(z_{n-1}\right)\right] / m^{n} \\
& \leq \mathbb{E}\left[w_{n-1}\right]+\mathbb{E}\left[z_{n-1}\right] G\left(\mathbb{E}\left[z_{n-1}\right]\right) / m^{n} \\
& \leq \mathbb{E}\left[w_{n-1}\right]\left\{1+G\left(m^{n-1}\right) / m\right\},
\end{aligned}
$$

where we used in turn the concavity of $x G(x)$, that $\mathbb{E}\left[z_{n-1}\right] \geq m^{n-1}$, and that $G$ does not increase. It follows that

$$
\mathbb{E}\left[w_{n}\right] \leq \prod_{k=1}^{\infty}\left\{1+G\left(m^{k}\right)\right\}<\infty,
$$

as $\sum G(k) / k<\infty \Leftrightarrow \sum G\left(m^{k}\right)<\infty$.

3. Markov branching. In continuous time the natural formulation of Markovian population size-dependent branching is to make the death intensity population size dependent, call it $\mu(n)$, and the same for the reproduction distribution, $p_{k}(n)$, as in the Galton-Watson case.

Since individuals do not age, there is complete symmetry between all those present in a population at the birth of any new member, and as in the Galton Watson case,

$$
\mathbb{E}\left[\frac{z_{t-\tau_{x}}(x)}{z_{t}} \mid \mathscr{G}_{x}\right]=\frac{1}{z_{\tau_{x}}}
$$

for all $t \geq \tau_{x}$. In other words:

LEMMA 7. Population size-dependent Markov branching processes grow homogeneously. 
Now write $\mu:=\mu(\infty), m(n):=\sum k p_{k}(n), m:=m(\infty)$ and introduce the corresponding Malthusian parameters $\alpha(n):=\mu(n)\{m(n)-1\}, n=1,2, \ldots, \infty$, $\alpha:=\alpha(\infty)$.

LEMMA 8. Assume that $\mu(n) \rightarrow \mu>0, m(n) \geq m$,

$$
\sum\{m(n)-m\} / n<\infty,
$$

and that for all $n$ and $k$,

$$
\mu(n) \sum_{i \geq k} p_{i}(n) \geq \mu \sum_{i \geq k} p_{i} .
$$

Then $\left\{\exp (-\alpha t) z_{t}, t \geq 0\right\}$ is tight.

Proof. With

$$
A_{t}:=\int_{0}^{t} \alpha\left(z_{u}\right) d u
$$

the random variables

$$
W_{t}:=\exp \left(-A_{t}\right) z_{t}, t \geq 0,
$$

constitute a nonnegative martingale with respect to its natural filtration. Hence, $W_{\infty}:=\lim _{t \rightarrow \infty} W_{t}$ exists almost surely and

$$
\mathbb{E}\left[W_{\infty}\right] \leq \lim _{t \rightarrow \infty} \mathbb{E}\left[W_{t}\right]=1
$$

However,

$$
\exp (-\alpha t) z_{t}=W_{t} \exp \left(A_{t}-\alpha t\right)=W_{t} \exp \left(\int_{0}^{t}\left\{\alpha\left(z_{u}\right)-\alpha\right\} d u\right) .
$$

The last assumption of the lemma serves to ensure that the population grows at least as fast as it would have done in an environment of an infinite population, $z_{u} \stackrel{d}{\geq} z_{u}^{\infty}$, if $z_{u}^{\infty}$ denotes the size of the latter population, that is, a Markov branching process defined by parameters $\mu$ and $p_{k}$ and no population size dependence. Since the latter grows as $\exp (\alpha u)$, if it does not die out (and only this case needs checking), it is enough that

$$
\int_{0}^{\infty}\{\alpha(c \exp (\alpha u))-\alpha\} d u
$$

converges or equivalently

$$
\int_{0}^{\infty}\{m(c \exp (\alpha u))-m\} d u<\infty
$$

However, that follows from the the first assumption, after a substitution of variable. 
THEOREM 9. Let $\left\{z_{t}\right\}$ be a continuous time, single type, population sizedependent Markov branching process, satisfying the $x \log x$-condition $\sum k$. $(\log k) p_{k}<\infty$ as well as the conditions of the preceding lemma. Then the process exhibits Malthusian growth, $z_{t} \sim \exp (\alpha t)$ on the set of nonextinction, as $t \rightarrow \infty$.

PROOF. It remains to check the convergence condition of Corollary 4. In terms of $g(n):=m(n)-m$ or rather Klebaner's (1989) $G \geq g$ of the preceding section,

$$
\begin{aligned}
\sum \mathbb{E}\left[g\left(z_{T_{i}}\right) / z_{T_{i}}\right] & \leq \sum \mathbb{E}\left[z_{T_{i}} G\left(z_{T_{i}}\right) / z_{T_{i}}^{2}\right] \\
& \leq \sum \mathbb{E}\left[z_{T_{i}} G\left(z_{T_{i}}\right)\right] \mathbb{E}\left[1 / z_{T_{i}}^{2} ; z_{T_{i}} \geq 1\right],
\end{aligned}
$$

since $z G(z)$ increases whereas $1 / z^{2}$ decreases. In the next step use that $z G(z)$ is concave and that $\mathbb{E}\left[z_{T_{i}}\right] \leq c i$ for any $c>m(1)-1$ in order to conclude that

$$
\mathbb{E}\left[z_{T_{i}} G\left(z_{T_{i}}\right)\right] \leq c i G(c i) .
$$

However, by a large deviations argument it is easy to check that for an $\varepsilon<$ $m-1$ (the superscript $\infty$ denoting a process in the environment of infinitely many individuals, $z_{t}^{\infty} \stackrel{d}{\leq} z_{t}$ ),

$$
\begin{aligned}
\mathbb{E}\left[1 / z_{T_{i}}^{2} ; z_{T_{i}} \geq 1\right] & \leq \mathbb{E}\left[1 /\left(z_{T_{i}}^{\infty}\right)^{2} ; z_{T_{i}}^{\infty} \geq 1\right] \\
& \leq \mathbb{E}\left[1 /\left(z_{T_{i}}^{\infty}\right)^{2} ; z_{T_{i}}^{\infty} \geq \varepsilon i\right]+\mathbb{P}\left(z_{T_{i}}^{\infty} \leq \varepsilon i\right) \leq C / i^{2} .
\end{aligned}
$$

The claimed convergence follows.

This theorem is again due to Klebaner (1994), with a.s. and $L^{2}$-convergence and a variance condition thus replacing the uniform $x \log x$ condition used in the Galton-Watson case. Note that the present theorem does not require the $x \log ^{2} x$. The first contribution to analysis of state-dependent Markov branching processes was Küster (1983).

4. Binary splitting with quiescence and aging Consider (population size-dependent) binary splitting processes, where cells either have i.i.d. cycle times (i.e., life spans) with a continuous distribution function $L$, ending with a mitotic division, or else they are quiescent, that is, remain and do not divide. Assume that cycling cells age in the sense that if $T$ is a typical cycle time, then

$$
\mathbb{P}(T>t+u \mid T>u)=\frac{1-L(t+u)}{1-L(u)} \leq 1-L(t)=\mathbb{P}(T>t) .
$$

Loosely speaking, death intensity increases with age. Population size dependence enters through the probability $p\left(z_{t}\right)$ with which a newborn cell embarks upon the cell cycle, otherwise turning quiescent. The population (tumor) size at $t$ is denoted by $z_{t}$. Again we consider the supercritical case: $p(n) \searrow p>1 / 2$, and refer to this process as population size-dependent supercritical splitting 
with aging. It is an age-dependent version of the Bell-Anderson cell sizedependent tumor model considered by Gyllenberg and Webb (1990).

LEMMA 10. The population size-dependent supercritical splitting process with aging displays homogeneous growth.

Proof. At each split one mother cell disappears and two new cells are added. Therefore, starting from one ancestor, $z_{\tau_{n}}=[n / 2]+1, n=1,2,3, \ldots$ as long as $\tau_{n}<\infty$. By the aging assumption the expected contribution from a newborn cell to the population later will not exceed that of an older cell. If $\mathscr{L}_{k}$ denotes the set of cells cycling at $\tau_{k}$, then by symmetry it is even true that

$$
\mathbb{E}\left[z_{\tau_{n}-\tau_{k}}\left(X_{k}\right) ; \tau_{n} \leq t<\tau_{n+1} \mid \mathscr{G}_{k}\right] \leq \mathbb{E}\left[z_{\tau_{n}-\tau_{k}}(x) ; \tau_{n} \leq t<\tau_{n+1} \mid \mathscr{G}_{k}\right]
$$

for any $x \in \mathscr{L}_{k}$. Hence,

$$
\begin{aligned}
& \mathbb{E}\left[\frac{z_{t-\tau_{k}}\left(X_{k}\right)}{z_{t}} \mid \mathscr{G}_{k}\right] \\
& \quad=\sum_{n \geq k} \mathbb{E}\left[\frac{z_{t-\tau_{k}}\left(X_{k}\right)}{z_{t}} ; \tau_{n} \leq t<\tau_{n+1} \mid \mathscr{G}_{k}\right] \\
& \quad=\sum_{n \geq k} \mathbb{E}\left[\frac{z_{\tau_{n}-\tau_{k}}\left(X_{k}\right)}{z_{\tau_{n}}} ; \tau_{n} \leq t<\tau_{n+1} \mid \mathscr{G}_{k}\right] \\
& \quad=\sum_{n \geq k} \frac{1}{[n / 2]+1} \mathbb{E}\left[z_{\tau_{n}-\tau_{k}}\left(X_{k}\right) ; \tau_{n} \leq t<\tau_{n+1} \mid \mathscr{I}_{k}\right] \\
& \quad \leq \sum_{n \geq k} \frac{1}{[n / 2]+1} \mathbb{E}\left[\frac{1}{z_{\tau_{k}}} \sum_{x \in \mathscr{L}_{k}} z_{\tau_{n}-\tau_{k}}(x) ; \tau_{n} \leq t<\tau_{n+1} \mid \mathscr{G}_{k}\right] \\
& \quad \leq \sum_{n \geq k} \frac{1}{[n / 2]+1} \mathbb{E}\left[\frac{z_{\tau_{n}}}{z_{\tau_{k}}} ; \tau_{n} \leq t<\tau_{n+1} \mid \mathscr{G}_{k}\right] \\
& \quad=\frac{1}{z_{\tau_{k}}} \sum_{n \geq k} \mathbb{P}\left(\tau_{n} \leq t<\tau_{n+1} \mid \mathscr{I}_{k}\right) \leq 1 / z_{\tau_{k}} .
\end{aligned}
$$

Define $\alpha$ as the Malthusian parameter of the limiting, infinite population,

$$
2 p \int_{0}^{\infty} \exp (-\alpha t) L(d t)=1
$$

If $\left\{z_{t}^{\infty}\right\}$ denotes an independent individual, binary splitting branching process, whose probability law is defined by $p$ and $L$, then clearly $\exp (-\alpha t) z_{t}^{\infty}$ has a non-trivial limit, as $t \rightarrow \infty$. Write $y_{t}$ for the total population at $t$, that is, all those born up to $t$ and let the superscript $c$ indicate that we only count cycling cells in $z_{t}^{c}$ or $y_{t}^{c}$.

LEMMA 11. The process $\left\{\exp (-\alpha t) z_{t}\right\}$ is tight, provided $\mathbb{E}[T]<\infty$ and $n\{p(n)-p\} \rightarrow 0$, as $n \rightarrow \infty$. 
PRoOF. Note that for any fixed $t_{0}, \exp (-\alpha t) z_{t} \leq y_{t_{0}}<\infty$ for $0 \leq t \leq t_{0}$. Therefore it is enough to check that lim $\sup \exp (-\alpha t) z_{t}$ is a finite random variable, and indeed since each quiescent cell had a cycling mother, that $\lim \sup \exp (-\alpha t) y_{t}^{c}$ is finite.

Define $\alpha_{n}$ by

$$
2 p(n) \int_{0}^{\infty} \exp \left(-\alpha_{n} t\right) L(d t)=1
$$

Then $\alpha_{n} \searrow \alpha$. It is easy to check that indeed

$$
0 \leq \alpha_{n}-\alpha \leq a\{p(n)-p\}
$$

for some $a>0$. For any individual $x$, let $b x$ denote its mother, $b^{2} x$ grandmother, and so on, $g x$ its generation and $T_{x}$ its life span (cell cycle duration). Define $r_{x}:=\alpha_{z_{\tau_{x}}}$ and write

$$
r_{n}=r_{X_{n}}=\alpha_{z_{\tau_{n}}}=\alpha_{[(n+2) / 2]}
$$

as before. Further, for any $x$, besides the ancestor, define

$$
A_{x}:=\sum_{k=1}^{g x} r_{b^{k} x} T_{b^{k} x} \quad \text { and } \quad A_{n}:=A_{X_{n}} .
$$

Adapted to varying Malthusian parameters, and considering only cycling cells, Nerman's (1981) martingale then takes the form

$$
R_{n}:=1+\sum_{k=1}^{n} \exp \left(-A_{k}\right)\left(\xi_{k} \exp \left(-r_{k} T_{k}\right)-1\right),
$$

where again somewhat cavalierly $T_{k}$ stands for $T_{X_{k}}$ and $\xi_{k}$ gives the number of cycling children of $X_{k}$. Since it is nonnegative, it has an almost sure limit, $R_{\infty}$. Following the approach from the paper quoted, $N(t, u)$, the number of births between $t$ and $t+u$ from mothers themselves born before $t$, satisfies

$$
N(t, u) \geq \sum_{k=1}^{y_{t}^{c}}\left\{\xi_{k} 1_{[0, u]}\left(T_{k}\right)-1\right\} \geq \sum_{k=1}^{y_{t}^{c}}\left\{\eta_{k} 1_{[0, u]}\left(T_{k}\right)-1\right\},
$$

the $\eta$ in place of $\xi$ indicating that some individuals may have been removed so that the limiting branching process is obtained. But the martingale $M_{t}:=R_{y_{t}^{c}}$ and the function $r(t):=r_{n}$ on $\tau_{n} \leq t<\tau_{n+1}$ in their turn satisfy

$$
M_{t} \geq \exp (-r(t)(t+u)) N(t, u) \geq \exp (-\alpha(t+u)) N(t, u) \exp \left(-\alpha(t+u) g\left(z_{t}\right)\right)
$$

in terms of $g(n):=p(n)-p$.

To investigate $(t+u) g\left(z_{t}\right)$, as $t \rightarrow \infty$ note that $z_{\tau_{n}}=[n / 2]+1$, whereas $\mathbb{E}\left[\tau_{n}\right] \leq n \mathbb{E}[T]$ on the set of growth (by induction, e.g.). Hence $\mathbb{E}\left[\tau_{n} g\left(z_{\tau_{n}}\right)\right] \rightarrow 0$ and the same must be true a.s. for $(t+u) g\left(z_{t}\right)$, as $t \rightarrow \infty$. By martingale 
convergence further $M_{t} \rightarrow R_{\infty}$ (on the set where $y_{t} \rightarrow \infty$ ), and by the law of large numbers,

$$
\sum_{k=1}^{y_{t}^{c}}\left\{\eta_{k} 1_{[0, u]}\left(T_{k}\right)-1\right\} / y_{t} \rightarrow 2 p L(u)-1>0
$$

for a suitable $u$. It follows that

$$
\begin{aligned}
& \lim \sup \exp (-\alpha t) y_{t}^{c} \\
& \quad \leq \lim \sup \exp (-\alpha t) y_{t}^{c} \frac{N(t, u)}{\sum_{k=1}^{y_{t}^{c}}\left\{\eta_{k} 1_{[0, u]}\left(T_{k}\right)-1\right\}} \\
& \quad \leq C \lim \sup \exp (-\alpha t) N(t, u) \leq C^{\prime} \lim \sup M_{t}=C^{\prime} R_{\infty}<\infty .
\end{aligned}
$$

Here $C$ and $C^{\prime}$ are positive constants.

The main result is rather direct from these lemmas and the basic Theorem 2 .

THEOREM 12. Let $\left\{z_{t}\right\}$ be a binary splitting population size-dependent process with aging and a continuous life length distribution $L$ with a finite mean, as defined. Assume that the probability of a newborn cell in an n-size population entering the cell cycle satisfies $p(n) \searrow p>1 / 2$ and $\sum\{p(n)-p\} / n<\infty$. Let $\alpha$ be the root of

$$
2 p \int_{0}^{\infty} \exp (-\alpha t) L(d t)=1
$$

Then $\exp (-\alpha t) z_{t}$ converges to a nonzero limit in probability on the set where the population does not die out.

Proof. With $\varepsilon_{i}=g\left(z_{\tau_{i}}\right)=p\left(z_{\tau_{i}}\right)-p$, we must only check that

$$
\sum_{i} \mathbb{E}\left[\varepsilon_{i} / z_{\tau_{i}}\right]<\infty
$$

However, this is clear since, as we have seen repeatedly, $z_{\tau_{i}}=[i / 2]+1$ on $\left\{\tau_{i}<\infty\right\}$.

5. Bellman-Harris-type processes. The crucial step in Lemma 10 was the monotonicity: when a process is delayed, splittings occur later and therefore the process tends to be smaller, provided the probability of dying without children is nil. This makes it plausible that results from the preceding section can be extended to general population size-dependent splitting processes with a probability $p_{k}(n)$ of begetting $k$ children if you die when population size is $n$, at least provided life spans are i.i.d., aging is there and $p_{0}(n)=0$ for all $n$.

We refer to such processes as population size-dependent Bellman-Harris processes and keep the notation from the preceding section wherever suitable. Without mentioning, we also assume that reproduction decreases in distribution with increasing population sizes (as in the Galton-Watson case) and that 
in the limit $p_{k}:=p_{k}(\infty)$, together with the continuous life span distribution $L$, defines a supercritical process with the Malthusian parameter $\alpha$.

LEMMA 13. Population size-dependent Bellman-Harris processes as above with aging and $p_{0}(n)=0$, for all $n$, exhibit homogeneous growth.

ProOF. Let $\mathscr{g}_{x}$ denote the set of individuals alive at $\tau_{x}$, besides $x$ herself. (We regard her mother but not possible sisters as dead.) Define a new branching population starting at $\tau_{x}$ by replacing the remaining life spans by i.i.d. $L$ life spans $\tilde{\lambda}_{x^{\prime}}, x^{\prime} \in \mathscr{F}_{x}$ but making no other changes. By the aging assumption the new initiating life spans are longer in distribution than the original ones, and we can construct them on a suitably enlarged probability space so that indeed $\tilde{\lambda}_{x^{\prime}} \geq \lambda_{x^{\prime}}-\tau_{x}+\tau_{x^{\prime}}$.

Let $\tilde{z}_{t}$ denote the resulting process, and write $\tilde{T}_{1}<\tilde{T}_{2}<\cdots$ for its successive splitting times after starting at $\tau_{x} . T_{1}, T_{2}, \ldots$ are the corresponding times in the original process. By construction $T_{i} \leq \tilde{T}_{i}$. Clearly the probability distribution for the number of children born in the original population at $T_{1}$ is the same as that ruling the number in the new population at $\tilde{T}_{1}$, namely $\left\{p_{k}\left(z_{\tau_{x}}\right)\right\}$. By induction, this equality of reproduction distributions holds also later and we can make the construction so that $z_{T_{i}}=\tilde{z}_{\tilde{T}_{\text {. }}}$ for all $i$.

However, since the processes $z_{t}$ and $\tilde{z}_{t}$ both increase, the postponed version must be smaller. We conclude that

$$
\mathbb{E}\left[z_{t-\tau_{x}}(x) / z_{t} \mid \mathscr{G}_{x}\right] \leq \mathbb{E}\left[z_{t-\tau_{x}}(x) / \tilde{z}_{t} \mid \mathscr{G}_{x}\right] .
$$

Yet, since $\tilde{z}_{t} \leq z_{t}$, the number of children being added to $x$ 's daughter process according to $\left\{p_{k}\left(z_{t-}\right)\right\}$ is smaller in distribution than if $\left\{p_{k}\left(\tilde{z}_{t-}\right)\right\}$ governs possible reproduction at $t$. We conclude that

$$
\mathbb{E}\left[z_{t-\tau_{x}}(x) / \tilde{z}_{t} \mid \mathscr{G}_{x}\right] \leq \mathbb{E}\left[\tilde{z}_{t-\tau_{x}}(x) / \tilde{z}_{t} \mid \mathscr{G}_{x}\right]=1 / z_{\tau_{x}}
$$

LEMMA 14. Consider a population size-dependent Bellman-Harris process with continuous life spans, $m(n) \searrow m>1$, and $\sum\{m(n)-m\} / n<\infty$. Then, in terms of the splitting times $T_{1}<T_{2} \ldots, \sum_{n} \mathbb{E}\left[\left(m\left(z_{T_{n}}\right)-m\right) / z_{T_{n}}\right]<\infty$.

The proof is as in the Markov case.

Finally, tightness follows as in Lemma 11.

THEOREM 15. Consider a Bellman-Harris process with population size dependence and i.i.d. continuously distributed life spans with aging and finite expectation. Denote the life span distribution by L. Assume that reproduction distributions, $\left\{p_{k}(n), k=1,2, \ldots\right\}$ if population size is $n$, distributionally majorize a reproduction $\left\{p_{k}, k=1,2, \ldots\right\}$ with mean $m>1$ and $\sum_{k} p_{k} k \log k<$ $\infty$, that $m(n) \searrow m$, and that

$$
\sum_{n}\{m(n)-m\} / n<\infty .
$$


Then, as $t \rightarrow \infty, \exp (-\alpha t) z_{t}$ has a nontrivial limit in probability for $\alpha$ the Malthusian parameter of the imaginary process in an infinite population environment, $m \int_{0}^{\infty} \exp (-\alpha t) L(d t)=1$.

Do not overlook that we have, alas, had to require that $p_{0}(n)=0$ for all $n$. It remains open to extend the symmetry argument from Lemma 10 to more general population processes.

Acknowledgement. Thanks are due to Mikael Andersson and Serik Sagitov for their helpful comments and to the referee who improved Theorem 5 through the reference to Dubuc's work.

\section{REFERENCES}

AthreyA, K. B. and Ney, P. (1972). Branching Processes. Springer, Berlin.

Dubuc, S. (1970). La fonction de Green d'un processus de Galton-Watson. Studia Math. 34 69-87.

GyllenberG, M. and WebB, G. F. (1990). A nonlinear structured cell population model of tumor growth with quiescence. J. Math. Biol. 28 671-694.

JAGERs, P. (1975). Branching Processes with Biological Applications. Wiley, Chichester.

JAGERs, P. (1989). General branching processes as Markov fields. Stochastic Process Appl. 32 $183-212$.

JAGERS, P. (1996). Towards dependence in general branching processes. In Classical and Modern Branching Processes (K. Athreya and P. Jagers, eds.) Springer, New York.

JAGERs, P. (1997). Coupling and population dependence in branching processes. Ann. Appl. Probab. 7 281-298.

Keller, G., Kersting, G. and Rösler, U. (1987). On the asymptotic behaviour of discrete time stochastic growth processes. Ann. Probab. 15 305-343.

Kersting, G. (1986). On recurrence and transience of growth models. J. Appl. Probab. 23 614625.

KLEBANER, F. (1984). Geometric rate of growth in population size dependent branching processes. J. Appl. Probab. 21 40-49.

KLEBANER, F. (1985). A limit theorem for population size dependent branching processes. J. Appl. Probab. 22 48-57.

KLEBANER, F. (1989). Geometric growth in near-supercritical population size dependent multitype Galton-Watson processes. Ann. Probab. 17 1466-1477.

KLEBANER, F. (1994). Asymptotic behaviour of Markov population processes with asymptotically linear rate of change. J. Appl. Probab. 31 614-625.

KÜsTER, P. (1983). Generalized Markov branching processes with state dependent offspring distribution. Z. Wahrsch. Verw. Gebiete 64 475-503.

KÜstER, P. (1985). Asymptotic growth of controlled Galton-Watson processes. Ann. Probab. 13 $1157-1178$.

Lindvall, T. (1992). Lectures on the Coupling Method. Wiley, Chichester.

NERMAN, O. (1981). On the convergence of super-critical general (C-M-J) branching processes. $Z$. Wahrsch. Verw. Gebiete 57 365-395.

Neveu, J. (1986). Arbres et processus de Galton-Watson. Ann. Inst. H. Poincaré 22 199-207.

School of Mathematical and Computing Sciences

Chalmers UNIVERSiTy OF TECHNOLOGY

AND GÖTEBORG UNIVERSITY

SE-412 96 GOTHENBURG

SWEDEN

E-MAIL: jagers@math.chalmers.se 